Document downloaded from:

http://hdl.handle.net/10251/64685

This paper must be cited as:

Redondo Foj, MB.; Sanchis Sánchez, MJ.; Ortiz Serna, MP.; Carsí Rosique, M.; García, JM.; García, FC. (2015). The effect of cross-linking on the molecular dynamics of the segmental and Johari-Goldstein processes in polyvinylpyrrolidone-based copolymers. Soft Matter. 11:7171-7180. doi:10.1039/c5sm00714c.

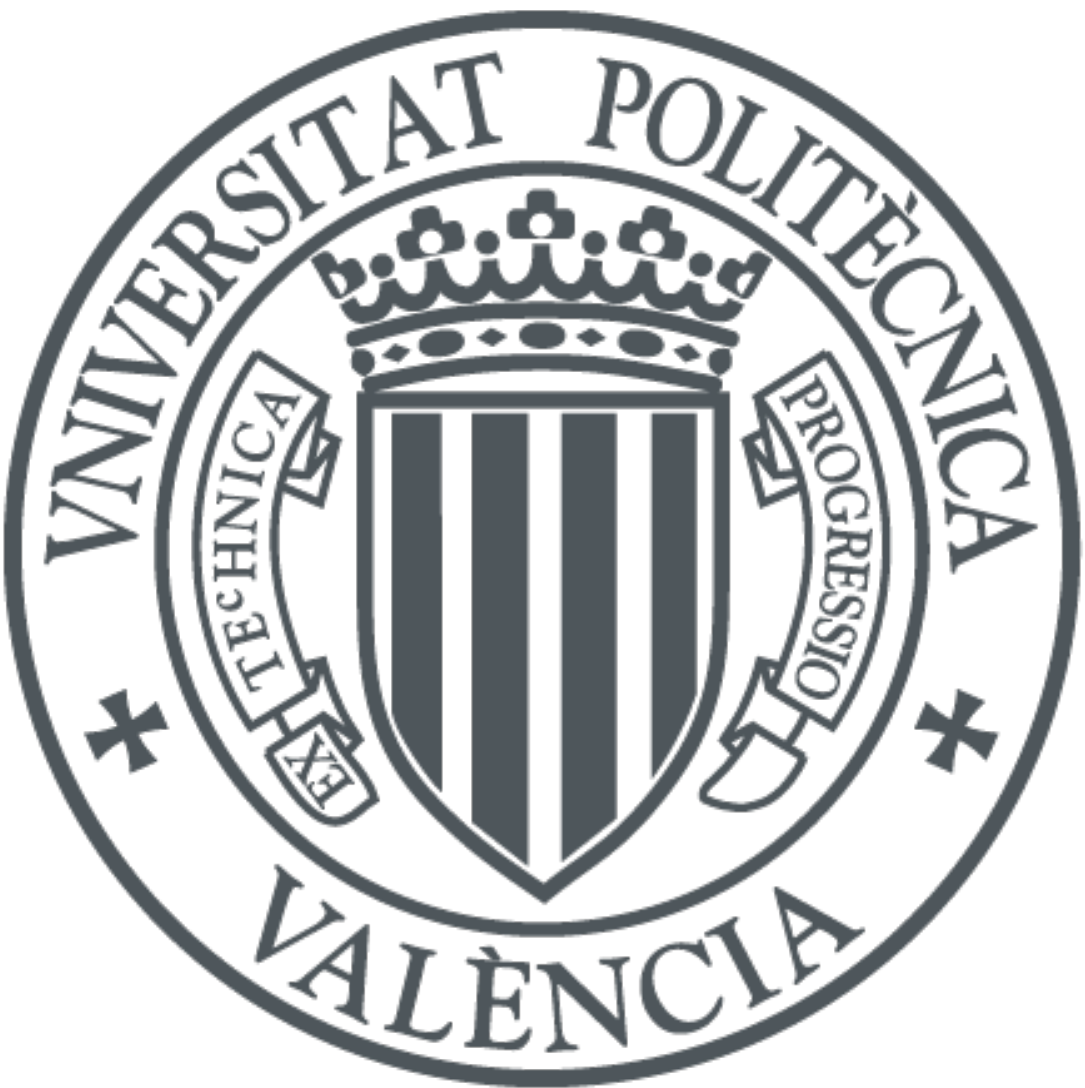

The final publication is available at

http://dx.doi.org/10.1039/C5SM00714C

Copyright Royal Society of Chemistry

Additional Information 


\section{Effect of cross-linking on the molecular dynamics of the segmental and $\beta$ Johari-Goldstein processes in Polyvinylpyrrolidone-based copolymers}

Belén Redondo-Foj ${ }^{a}$, María Jesús Sanchis $^{\mathrm{a}}$, Pilar Ortiz-Serna ${ }^{\mathrm{a}}$, Marta Carsí ${ }^{\mathrm{b}}$, José Miguel García ${ }^{c}$ and Felix Clemente García ${ }^{c}$

The effect of the cross-link density on the molecular dynamics of copolymers composed of vinylpyrrolidone (VP) and butyl acrylate (BA) was studied using differential scanning calorimetry (DSC) and dielectric relaxation spectroscopy (DRS). A single glass transition was detected by DSC measurements, showing conolymer miscibility. The dielectric spectra exhibit conductive processes and three dipolar relaxations labeled as $\alpha, \beta$ and $\gamma$ in decreasing order of decreasing temperatures. The cross-linker content affects both $\alpha$ and $\beta$ processes, but the fastest $\gamma$ process is relatively unaffected. An increase of the cross-linking produces a typical effect on the $\alpha$ process dynamics: (i) the glass transition temperature is increased, (ii) the dispersion is broadened (iii) its strength is decreased and (iv) the relaxation times are increased. However, the $\beta$ process, which is a Johari-Goldstein relaxation-possesses typical features of pure Johari-Goldstein relaxation, unexpectedly loses the intermolecular character for the highest cross-linker content.

\footnotetext{
- Instituto Tecnología Eléctrica, Departamento de Termodinámica Aplicada, Universitat Politècnica de València, Cami de Vera $s / n, 46022$, Valencia, Spain. Correspondence to: Belen Redondo-Foj (E-mail: marefo@ite.upv.es)

b. Instituto de Automática e Informática Industrial, Universitat Politècnica de

València, Camí de Vera $s / n, 46022$, Valencia, Spain

Universidad de Burgos, Plaza Misael Bañuelos s/n, 09001 Burgos, Spain
} 


\section{Introduction}

Polyvinylpyrrolidone (PVP) is widely used in a number of industrial fields, such as biomedical and pharmacological applications due to its biocompatibility, adhesives, ceramic, coatings, food, etc. ${ }^{1,2}$ However, PVP cannot be melt processed because of its low decomposition temperature. Moreover, its films prepared by casting (from PVP solutions) or by bulk polymerization (crosslinked PVP) are brittle. These drawbacks can be overcome by copolymerization. The copolymerization is shown as an effective method to prepare macromolecules with specific chemical structures and to control some properties such as hydrophilic/hydrophobic balances, solubility, polarity, etc. ${ }^{3}$ Copolymers comprised of vinylpyrrolidone (VP) and acrylic or vinyl comonomers are produced to modify, to improve and to adapt the properties of PVP to specific applications. ${ }^{4-7}$ Thus, acrylic chemicals are among the most versatile and inexpensive comonomers to prepare materials with specific properties. In particular, butyl acrylate (BA) is presented as a valuable acrylic comonomer for tuning the properties of PVP. The homopolymer from BA (PBA) is hydrophobic and has a glass transition of 219

$\mathrm{K}$, while the PVP is an extremely hydrophilic polymer (uncross-linked PVP is water soluble), with glass transition of $448 \mathrm{~K}$. Thus, copolymers comprised of VP and BA can be presented as tractable materials with moderate hydrophilicity and good mechanical and thermal properties.

In addition to the copolymers, it is also highly interesting the study of cross-linked polymer networks. The dynamics of cross-linked polymers is influenced by the network composition and the polymer chain architecture. As it is well-known, both the glass-rubber and the subglass transitions are highly sensitive to changes in the backbone structure, the cross-link density, and the introduction of pendant groups or branches. As a result, the relationship between network structure, chain dynamics and bulk properties of cross-linked polymers has been widely studied in the literature using different techniques for a wide range of material systems. ${ }^{8-13}$ In particular, the dielectric relaxation spectroscopy (DRS) is presented as one of the most powerful techniques to study the chain dynamics of solid polymers with temperature and frequency. This technique enables the analysis of the chains response over more than 10 decades in the frequency domain. ${ }^{14}$

As previously reported, the introduction of network junctions due to cross-linking is reflected in the increase in the glass transition temperature $\left(T_{\partial}\right) \cdot{ }^{15-16}$ The effect of cross-linking in the segmental transition, also called $\alpha$ relaxation, usually broadens the dispersion and makes its relaxation time more sensitive to temperature, that is, the material becomes more fragile. On the other hand, it is found that the cross-linking may produce different effects in the sub-glass transitions, which may be related to their molecular origin. ${ }^{17-23}$ Thus, the secondary relaxations can have (i) an intramolecular origin, which involves the independent motion of an isolated group or (ii) an intermolecular origin, which involves the motion of essentially the entire molecule (repeat unit in the case of a polymer). The latter are known as Johari-Goldstein (JG) relaxations. ${ }^{24-26}$ However, only from the dielectric relaxation data, it is difficult to know whether the secondary relaxations have an intra- or intermolecular origin. To overcome this difficulty, Ngai et al proposed the comparison of the JG secondary relaxation time, $\tau_{J G}$, and the primitive (non-cooperative) relaxation time, $\tau_{0}$, of the Coupling Model $(\mathrm{CM}){ }^{26-31}$. The basis of the $\mathrm{CM}$ is the existence of a temperature-insensitive crossover time, $t_{c}$, which is about 2 ps for small molecules and polymeric glass formers ${ }^{32,33}$. At times shorter than $t_{c}$, there are basic molecular units that relax independently of each other, whose relaxation function can be described by a simple exponential decay:

$$
\left.\phi()_{t}\right)=\exp _{t}[-(\tau)], t<t_{c}
$$

However, at times longer than $t_{c}$, the intermolecular interactions (i.e. cooperativity ${ }^{34}$, which is associated with the dynamic heterogeneity) slow the relaxation. Thus, there is a coupling between the motions of the different basic molecular units. Therefore, the relaxation function assumes the Kohlrausch-Williams-Watts (KWW) equation $^{35}$ :

$\phi(t)=\exp \left[-\left(t / \tau^{*}\right)^{\beta_{k w w}}\right], t<t$

where $\tau^{*}$ is the $\alpha$ relaxation time, i.e. $\tau^{*}=\tau_{\alpha}$ and $\beta_{K w w}$ parameter is the stretch exponent that lies in the range 01 and quantifies the extent of deviation from pure exponentially, i.e. the degree of the relaxation time dispersion. The case $\beta_{k w w}=1$ refers to simple exponential behaviour. The coupling parameter $n$, which characterizes the strength of the intermolecular constraints on segmental relaxation, can be evaluated as $n=\left(1-\beta_{K w w}\right)$.

Continuity of $\phi(t)$ at $\mathrm{t}=t_{c}$ leads to the relation between the two relaxation times:

$\tau_{\alpha}=\left[t_{c}^{\beta_{k W w}-\tau_{0}}\right]^{1 / \beta}{ }_{k w w}, t=t_{c}$

Thus, a secondary relaxation is considered a JG relaxation when its relaxation time, $\tau_{\beta}$, obeys the following criterion $^{36}$ :

$\tau_{\beta}=\tau_{J G} \approx \tau_{0}$

This relation has been fulfilled for genuine JG 
relaxations in many glass formers. ${ }^{28,37}$ At any temperature, the primitive relaxation time can be calculated by the CM using the Eq. (3) that relates $\tau_{0}$ to the $\alpha$ relaxation time, $\tau_{\alpha}$, as follows:

$$
\tau_{0}=t_{c}^{n} \cdot\left(\tau_{\alpha}\right)^{1-n}
$$

In previous works, we studied the effect of copolymer composition in a family of vinylpyrrolidone (VP) and butyl acrylate (BA) copolymers with a different proportions of monomers and a fixed cross-linker molar ratio of $1 \%$, using different characterization techniques. ${ }^{38-39}$ The dielectric spectra showed three relaxations, $\gamma, \beta$ and $\alpha$, followed by conductive processes. It was observed dipole-dipole interactions between the lactam groups of VP, which was found to exert an important influence in the molecular mobility. A single $T_{g}$ was detected by DSC measurements showing the copolymer miscibility, and it was found to be strongly dependent of the composition. The $\gamma$ process was related to local motions while the $\beta$ process was assigned to a JG process.

In this paper, we examine the effect of the crosslinking on the dynamics of 60VP/40BA copolymer. This copolymer ratio was chosen because this sample has the most balanced properties compared with other compositions. $^{39}$ The study is mainly focused on the segmental and the JG processes due to their intermolecular character. A detailed comparative analysis between the samples with different cross-link densities is carried out.

\section{Experimental}

\section{Sample preparation}

The molar ratio of the comonomers 1-vinyl-2-pyrrolidone (VP), butyl acrylate (BA) was 60/40. The synthesis procedure was previously described elsewhere, along with the discussion concerning the random copolymerization of VP and $\mathrm{BA}_{38}$ (mononomer reactivity ratios: $r_{v P}=0.12$ and $\left.r_{B A}=0.58\right)$. The cross-linker used was the ethylene glycol dimethacrylate (C). Cross-linker molar ratios of $Z=$ $0.2,1$ and $2 \%$ were used in order to evaluate the influence of the cross-link density on the 60VP/40BA-ZC sample properties (see Fig. 1).
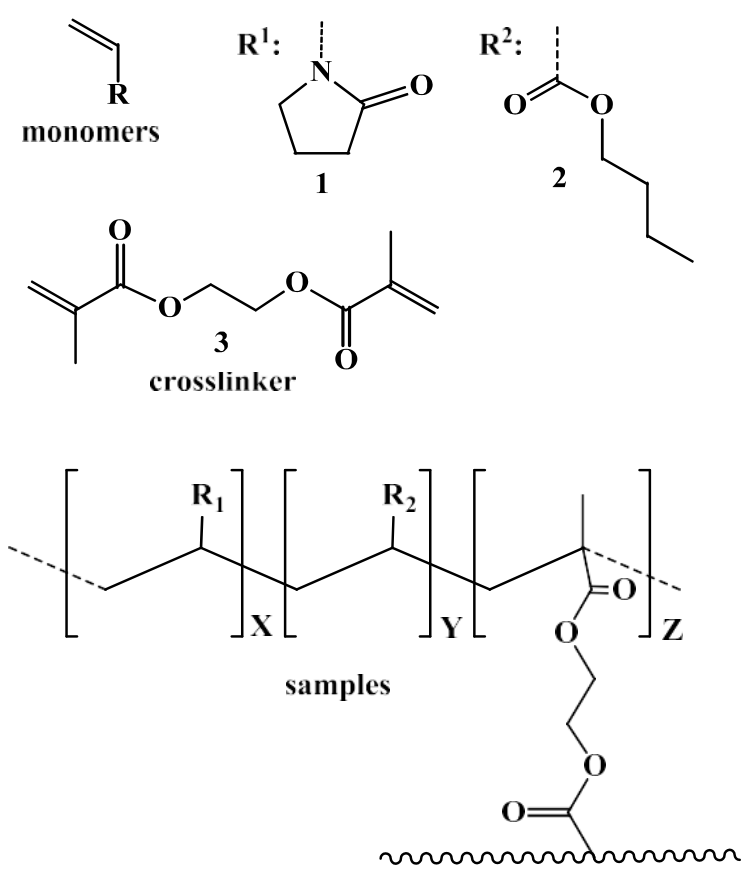

Fig. 1 Scheme of the chemical structure of 60VP/40BA-ZC samples, where $X=60$ and $Y=40$.

In order to characterize the chemical composition of the samples, Fourier Transform Infrared (FTIR) measurements were carried out. As described in a previous work $^{39}$, the FTIR spectra showed the characteristic absorption bands corresponding to the majority pendant lactam structure (lower energy band) and to the pendant butyl ester structure together with the cross-linker agent (higher energy band). However, the spectra did not show significant differences with the increase of cross-link density.

\section{Modulated Differential Scanning Calorimetry Measurements (MDSC)}

MDSC of 60VP/40BA-ZC samples was carried out using a TA Instruments DSC Q-20 with a refrigerated cooling system. The DSC tests were performed under a $50 \mathrm{ml} \cdot \mathrm{min}^{-1}$ flow of nitrogen to prevent oxidation. High-purity indium was used to calibrate the cell. The thin films were repeatedly stacked into a pan, with a weight of approx. 9.0 $\mathrm{mg}$. The measurements were conducted in crimpled nonhermetic aluminium pans, using an empty pan as the reference cell. The standard modulation conditions used in the experiments were $1 \mathrm{~K}$ of amplitude and $60 \mathrm{~s}$ of period. Each measurement was taken in two cycles from 193K to $473 \mathrm{~K}$ at a heating rate of $3 \mathrm{~K} \cdot \mathrm{min}^{-1}$. The first run was carried out in order to remove all the residual moisture and the thermal and mechanical history. The $T_{g}$ was evaluated as 
the intersection of the base line of the glassy region with the tangent to the endotherm in the middle point.

\section{Mechanical properties}

The tensile properties of the 60VP/40BA-ZC samples were analyzed using a Hounsfield H10KM Universal Testing Dynamometer. Strips were cut from the polymer films with $5 \mathrm{~mm}$ of width, $30 \mathrm{~mm}$ of length, and 100-120 $\mu \mathrm{m}$ of thick. The samples were dried at $363 \mathrm{~K}$ for $1 \mathrm{~h}$ and then measured at $303 \mathrm{~K}$ with an extension rate of 5 or 10 $\mathrm{mm} \cdot \mathrm{min}^{-1}$ and a gauge length of $10 \mathrm{~mm}$. At least six samples were tested for each polymer in order to average the data obtained.

\section{Dielectric Relaxation Spectroscopy (DRS)}

Isothermal relaxation spectra of 60VP/40BA-ZC samples were collected by using a Novocontrol Broadband Dielectric Spectrometer (Hundsagen, Germany) consisting of an Alpha analyser to carry out measurements from 5.10 ${ }^{2}$ to $3 \cdot 10^{6} \mathrm{~Hz}$. The measurements were performed in inert $\mathrm{N}_{2}$ atmosphere from $423 \mathrm{~K}$ to $123 \mathrm{~K}$. The temperature was controlled by a nitrogen jet (QUATRO from Novocontrol) with a temperature error of $\pm 0.1 \mathrm{~K}$ during every single sweep in frequency. Moulded disc shaped samples of about $0.12 \mathrm{~mm}$ thickness and $20 \mathrm{~mm}$ diameter were used. Glass fibre spacers were used to ensure the stability of the samples thickness at high temperatures. In order to avoid the increase of conductivity due to the water, the samples were previously dried at $343 \mathrm{~K}$ in a vacuum oven until constant weight. The experimental uncertainty was better than $5 \%$ in all cases.

\section{RESULTS AND DISCUSSION}

\section{MDSC Measurements}

The DSC thermograms (reversing curves) obtained for all the samples are plotted in Fig. 2, and their characteristic temperatures $\left(T_{g}, T_{\text {on }}, T_{\text {end }}\right)$ and the associated Heat Capacity changes $\left(\Delta C_{p}\right)$ are summarized in Table 1.

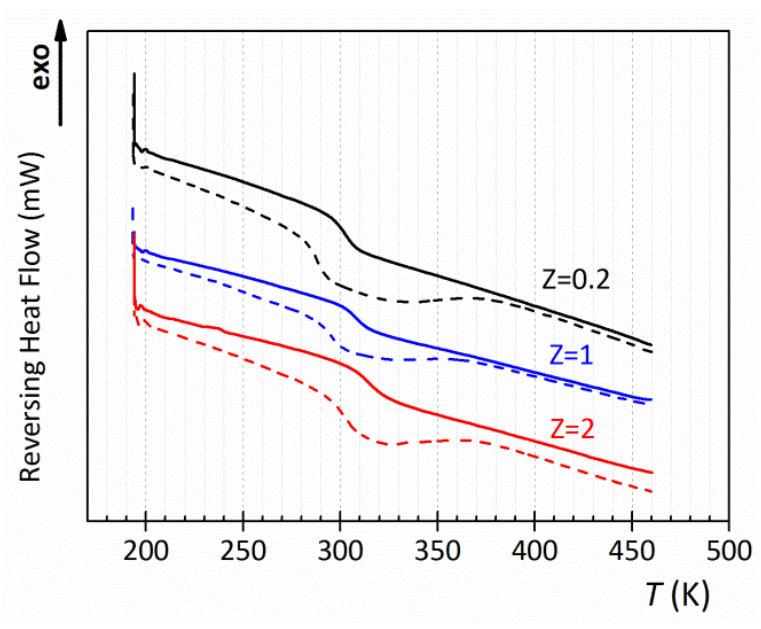

Fig. 2 MDSC thermograms (reversing curves) for 60VP/40BA-ZC samples: (i) $1^{\text {st }}$ heating ramp (dashed lines) and (ii) $2^{\text {nd }}$ heating ramp (continuous lines). Data are vertically shifted for a better visualization.

All DSC thermograms present a first step related to the glass rubber transition temperature. A single $T_{g}$ is observed showing the copolymer miscibility. This first step is well-defined in both the first and the second reversing curves but appears at lower temperatures in the first heating ramp, due to the plasticizing effect of water. On the other hand, it is observed an increase of the $T_{g}$ value of about $12 \mathrm{~K}$ with the increase of cross-linking. This is due to the decrease in the mobility imposed by the cross-links to segmental motion. Moreover, the width of the glass transition ( $\Delta T=T_{\text {end }}-T_{\text {on }}$ ) also increases with the cross-linker content. $\Delta T$ parameter gives a qualitative indication of the relative homogeneity of the system because it may reflect the magnitude of local compositional fluctuations. ${ }^{40}$ Hence, this broadening is due to an increase in small-scale compositional fluctuations in the copolymer related to the cross-linking. Finally, the constraints imposed by the crosslinks also results in a decrease in $\Delta C_{p}\left(T_{g}\right)$, related to the presence of orderly packing of polymer molecules, which lowers the mobility at $T_{g} \cdot{ }^{39,41,42}$ Moreover, the $\Delta C_{p}$ value may include enthalpy contribution from the breaking of dipole-dipole bonds during heating.

\section{Mechanical properties}

It is well known the relationship between the mechanical properties and the chemical composition of every material. As it is expected, the increase of the cross-linker content, from $Z=0.2$ to $Z=2(60 \mathrm{VP} / 40 \mathrm{BA}-\mathrm{ZC})$ ), involves a reduction in the elongation, along with a concomitant hardening of the materials showing by the increase of the Young's modulus (Table 2). 
Table 1 Characteristic Temperatures and Heat Capacity Change of 60VP/40BA-ZC ( $Z=0.2,1$ and 2$)$ samples.

\begin{tabular}{lccc}
\hline Sample & $\mathrm{Z}=0.2$ & $\mathrm{Z}=1$ & $\mathrm{Z}=2$ \\
\hline$T_{g}^{\text {MDSC }}(K)$ & 302.4 & 308 & 314.6 \\
$T_{\text {on }}(K)$ & 296.0 & 301.8 & 308.3 \\
$T_{\text {end }}(K)$ & 307.9 & 314.9 & 322.9 \\
$\Delta T(K)$ & 11.9 & 13.1 & 14.6 \\
$\Delta C_{p}(J / g K)$ & 0.201 & 0.163 & 0.158 \\
$\# T_{g}^{\text {DRS }}(K)$ & 291.5 & 298.6 & 310.5 \\
$T_{g}^{\text {DRS }}(K)$ evaluated at $\tau=100 \mathrm{~s}$ & & \\
\hline
\end{tabular}

Table 2 Thickness and mechanical properties of the 60VP/40BA copolymer as a function of the cross-linker agent content.

\begin{tabular}{|c|c|c|c|}
\hline Sample & $Z=0.2$ & $Z=1$ & $Z=2$ \\
\hline sample thickness $(\mu \mathrm{m})$ & 115 & 115 & 115 \\
\hline Young modulus" (MPa) & $187^{b}$ & $200^{a}$ & $286^{a}$ \\
\hline Tensile strength ${ }^{\#}(\mathrm{MPa})$ & $15^{b}$ & $15^{a}$ & $19^{a}$ \\
\hline Elongation ${ }^{\#}(\%)$ & $900^{b}$ & $540^{a}$ & $390^{a}$ \\
\hline sample thickness $(\mu \mathrm{m})$ & 115 & 115 & 115 \\
\hline \multicolumn{4}{|c|}{${ }^{\#}$ Extension rate of $5^{a}$ or $10^{b} \mathrm{~mm} \cdot \mathrm{min}^{-1}$} \\
\hline
\end{tabular}

\section{DRS measurements}

A detailed analysis of the sample with $1 \%$ of cross-linked (60VP/40BA-1C) was previously reported by our research group. ${ }^{38}$ For this copolymer, at high temperatures and low frequencies the conductive contributions overlapped the $\alpha$-relaxation. The $\beta$ process was found to be a JG secondary relaxation and it was related to the local motions of the pyrrolidone group accompanied by the motion of the segments of the polymer backbone. The $\gamma$ process was connected with the butyl unit's motions located in the side chains of the polymer.
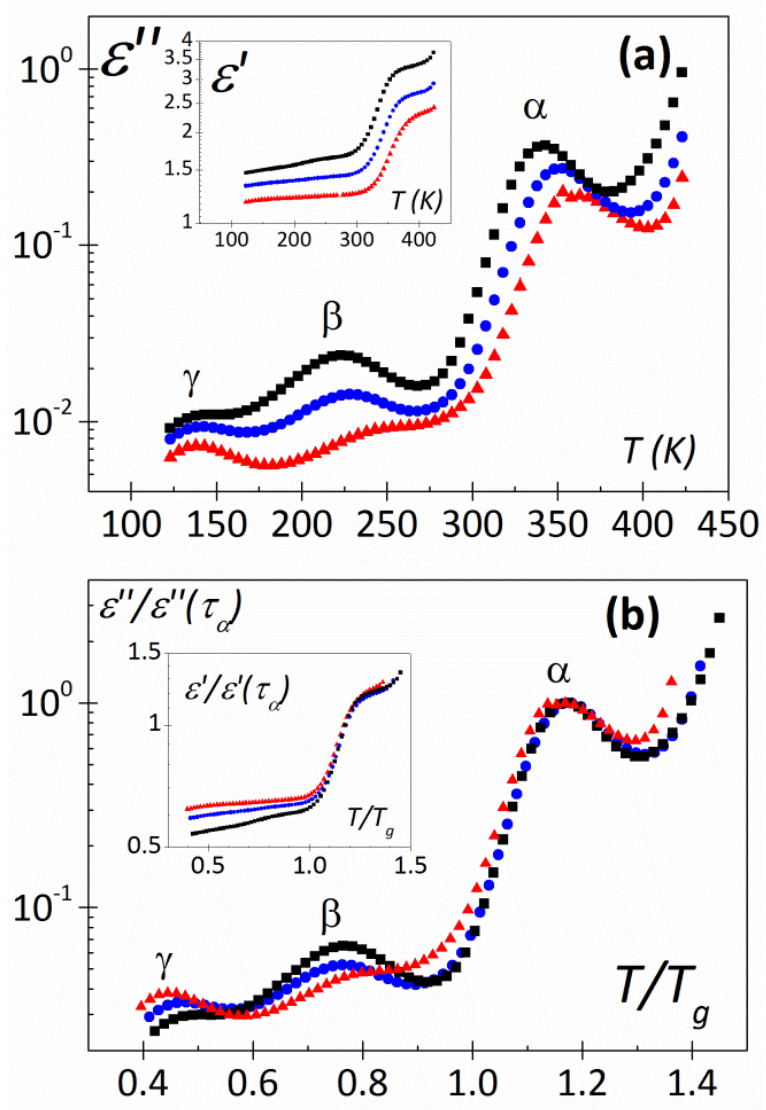

Fig. 3 (a) Temperature dependence of the dielectric and loss permittivity at $1.13 \cdot 10^{4} \mathrm{~Hz}$ for 60VP/40BA-ZC samples with $Z=0.2$ (square), $Z=1$ (circle) and $Z=2$ (triangle). (b) Temperature dependence of the normalized permittivity at $1.13 \cdot 10^{4} \mathrm{~Hz} \quad\left(\varepsilon^{\prime \prime}\left(\tau_{\alpha}\right)\right.$ is the permittivity at the characteristic time ( $\left.\tau_{\alpha}=100 \mathrm{~s}\right)$ for $\alpha$ relaxation).

In the present study, we have focused on the analysis of the cross-linker content effect on the dynamic mobility of the 60VP/40BA copolymer. For this purpose we have analysed other two 60VP/40BA-ZC copolymers with a lower $(Z=0.2)$ and a higher $(Z=2)$ cross-linker content.

Fig. 3a shows the temperature dependence of the dielectric permittivity [inset of Fig. 3a] and loss factor, at $1.1310^{4} \mathrm{~Hz}$, for the three samples analysed. As it is observed, the position of the peaks shifts to higher temperatures with the increase of cross-linker content and the relaxation curves tend to broaden. This shift indicates the increase of the $T_{g}$ with the cross-link density. In addition, a decrease in the intensity of the loss peaks is observed as the cross-linking increases. It is especially remarkable the case of $\beta$-relaxation, whose intensity decreases significantly with the cross-linking. In a previous work $^{18}$, we also observed a significant effect of the crosslinking on the $\beta$ relaxation. In that work, when the poly(2ethoxyethyl methacrylate) was cross-linked using $2.5 \%$ 
(mol) of ethylene glycol dimethacrylate, the $\beta$ peak completely disappeared in the spectra.

On the other hand, in order to establish a better comparison of the samples, the normalized curves are also represented in Fig. 3b. Qualitatively, the growth in the breadth indicates a broader distribution of relaxation times. It is also observed a significant increase of the loss factor at high temperatures that is associated with the conductivity contribution. The conductive processes can be originated not only by the migration of mobile charge carriers across the medium, but also by the trapping of charges at interfaces and boundaries. Thus, this additional polarization is the result of (i) accumulation of charges at the electrode-sample interface, called "electrode polarization" (EP) ${ }^{14}$ and/or (ii) the separation of charges at internal phase boundaries referred to as Maxwell-WagnerSillars (MWS) polarization. ${ }^{43,44}$

In order to obtain a better visualization of the effect of the cross-linker content on the conductive processes, it is advisable to use other formalisms for the data representation: (i) the dielectric loss modulus $M^{\prime \prime}(\omega)$ evaluated from $M^{*}(\omega)=M^{\prime}(\omega)+i M^{\prime}(\omega)=1 \varepsilon^{*}(\omega){ }^{45}$, where $\omega=2 \pi f$ is the angular frequency, (ii) the $\tan \delta(\omega)=\varepsilon^{\prime \prime}(\omega) \varepsilon^{\prime}(\omega)$ and (iii) the real part of the complex conductivity, $\sigma^{\prime}(\omega)=\omega \varepsilon_{0} \varepsilon^{\prime \prime}(\omega)$, since the EP and MWS contributions are better visualized. The normalized isochrones of $\varepsilon^{\prime \prime}, M^{\prime \prime}$, tan $\delta$ and $\sigma^{\prime}$ at $75 \mathrm{~K}$ above $T_{g}$ for the three samples are represented in Fig. 4 . In the low frequency zone, the sample with the highest crosslinker content shows a more complicate spectrum. Thus, the slope of the loss factor is reduced, whereas the breadth of the loss modulus peak is increased with the cross-linker content. On the other hand, two overlapping conductive processes appear in the spectrum when the dielectric data are plotted in terms of $\tan \delta$. The same effect is visualized when the real part of the complex conductivity is represented. In Fig. 4, the high frequency conductive process is noted as MWS. This process is generally evident in non-homogenous materials like multiphase polymers, blends and colloids, crystalline or liquid crystalline polymers, composites, etc. and occurs across smaller size scales when comparing to the electrode polarization. The MWS effects are more pronounced for conductive materials and, in certain cases, this large-scale polarization can mask the dielectric orientation response of the material.

Although the Kohlrausch-William-Watts (KWW) ${ }^{46-47}$ and the Havriliak-Negami $(\mathrm{HN})^{48-50}$ formalisms are equally able to fit the experimental data for the uncross-linked copolymer, the $\mathrm{HN}$ formalism provided superior fits at high levels of cross-linking. ${ }^{43}$ For this reason, the analysis of the dielectric spectra was made in the frequency domain using the empirical equation of $\mathrm{HN}$, which relates the complex permittivity $\left(\varepsilon^{*}\right)$ to the frequency by

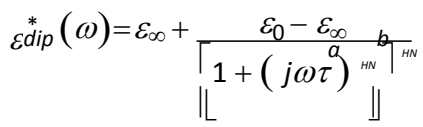

where the subscripts $\varepsilon_{0}$ and $\varepsilon_{\infty}$ are, respectively, the relaxed $(\omega=0)$ and unrelaxed $(\omega=\infty)$ dielectric permittivity, and $\tau$ is the relaxation time of the process. The dielectric strength, defined as $\Delta \varepsilon=\varepsilon_{0}-\varepsilon_{\infty}$, is related to both the dipole moment and the number of entities that participate in the relaxation process. The shape parameters $a_{H N}$ and $b_{H N}$ fulfil the condition $0<a_{H N} b_{H N} \leq 1$, and for a Debye process $a_{H N}=b_{H N}=1$. The $a_{H N}$ parameter is related to the departure of the complex $\varepsilon^{\prime \prime} v s \varepsilon^{\prime}$ plot from a semi-circumference at low frequencies, the lower the $a_{H N}$ parameter, the higher the departure is. In the $\varepsilon^{\prime \prime}$ spectrum, this parameter is related to the peak width, the higher the $a_{H N}$ parameter the narrower the peak is. The $b_{H N}$ parameter is related to the skewness of the plot along a straight line at high frequencies. For secondary absorptions, the $b_{H N}$ parameter is equal to one. The $\mathrm{HN}$ fit was carried out in two different zones in order to facilitate the characterization of the dipolar and conductive processes. As an example, the deconvolution procedure is depicted in Fig. 5 for two isothermals, one of them in the high temperature zone (conductivity and $\alpha$ processes) and the other one in the low temperature zone ( $\beta$ and $\gamma$ processes). 


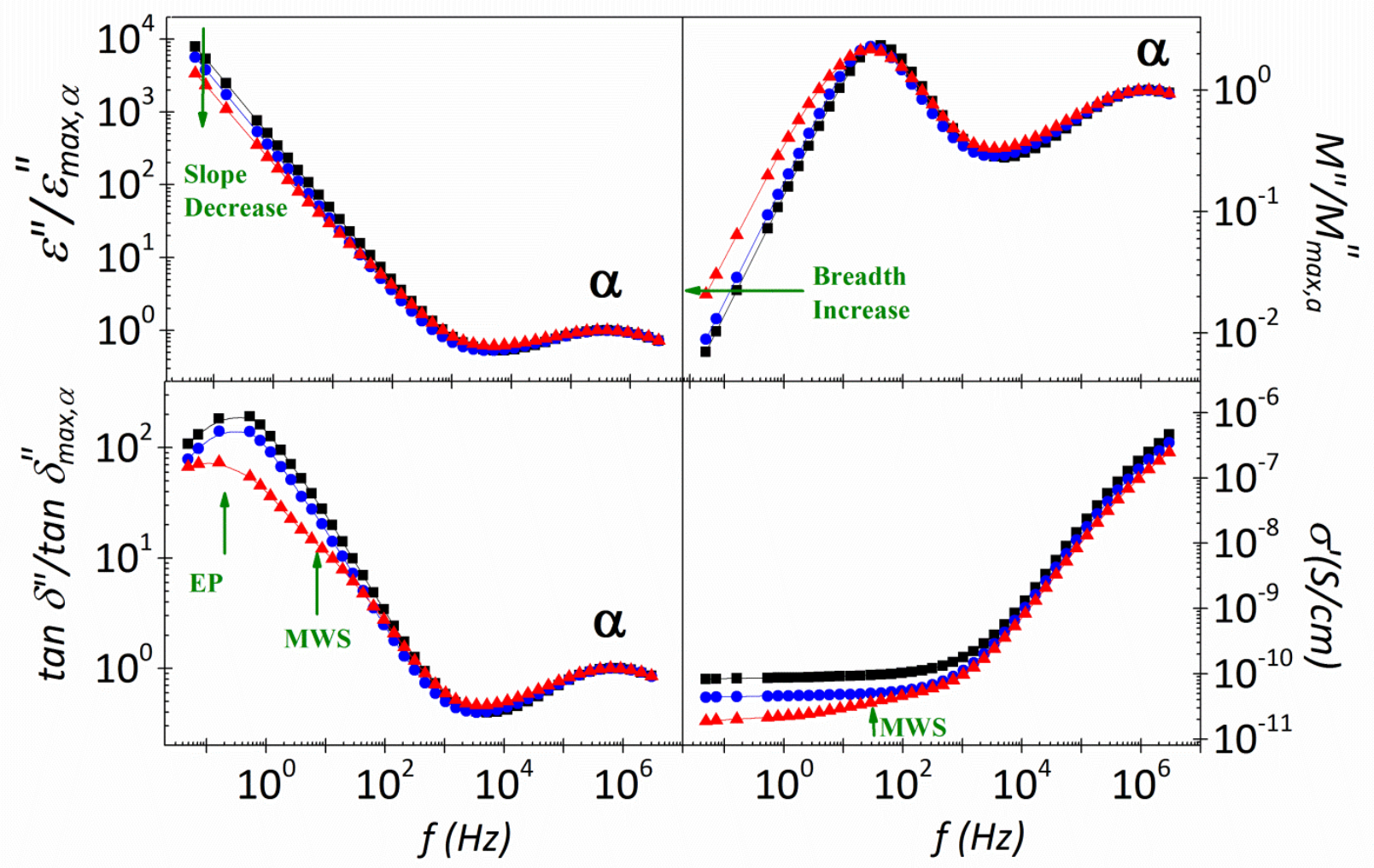

Fig. 4 Frequency dependence of $\varepsilon^{\prime \prime}, M^{\prime \prime}$, tan $\delta$ and $\sigma^{\prime}$ at $\left(T_{g}+75 K\right)$ for 60VP/40BA-ZC samples with Z=0.2 (square), Z=1 (circle) and Z=2 (triangle).

In the low temperature zone, an additive rule for the permittivity was assumed for the analysis of the dielectric loss spectra of the secondary processes. ${ }^{34}$

$$
\varepsilon_{\text {dip }}^{*}(\omega)=\operatorname{lmag} \mid \sum_{\mid i=1}^{2} \frac{\Delta \varepsilon_{i}}{\| 1+(j \omega \tau)^{q, H N} \prod_{i, H N} \mid}
$$

The HN parameters of the two secondary relaxations were determined at several temperatures from a multiple non-linear regression analysis of the experimental data. The four characterizing peak parameters $\left(\Delta \varepsilon_{i}, \tau_{i}, a_{H N, i}, b_{H N, i}\right)$ were allowed to vary and the obtained values are plotted in Figs. 6 to 8 .

In the high temperature zone, where the conductive process is dominant, we have include्d a neww term in order to characterize the spectra: $\varepsilon^{*}(\omega)=\varepsilon_{\text {dip }}+\varepsilon_{\text {cond }}$ with $\varepsilon_{\text {cond }}^{*}(\omega)=j\left(\begin{array}{c}\sigma \rho \omega^{s} \\ 0\end{array}\right)$ where $e_{0}$ is the permittivity of free space $\left(e_{0}=8.854 \mathrm{pF} \cdot \mathrm{m}^{-1}\right), \sigma$ is the conductivity arising from charges transport at the liquid-electrode interface and $s$ is a constant $(s \leq 1)$, which was approximately one for all the samples. For the $Z=2$ sample, it was necessary to limit the fitting parameters due to the presence of MWS process at intermediate frequencies between the $\alpha$ and conductivity processes. However, it was not possible to characterize the MWS process because it is not clearly defined in our spectra (see Fig. 4).

The temperature dependence of the relaxation strength, for all relaxation processes, is shown in Fig. 6. For $\beta$ and $\gamma$ processes this dependence follows the classical trends, that is, the dielectric strength increase or nearly remain constant with the temperature. This fact is consistent with a thermally activated mechanism because the dipole mobility rises with the temperature, associated with the free volume increase. Non-significant changes in the dielectric strength for the $\gamma$ absorption with the cross-linker content are observed. However, for the $\beta$ absorption a significant reduction in the relaxation strength is observed for the samples with a $Z=1$ and $Z=2$ cross-linker content. 

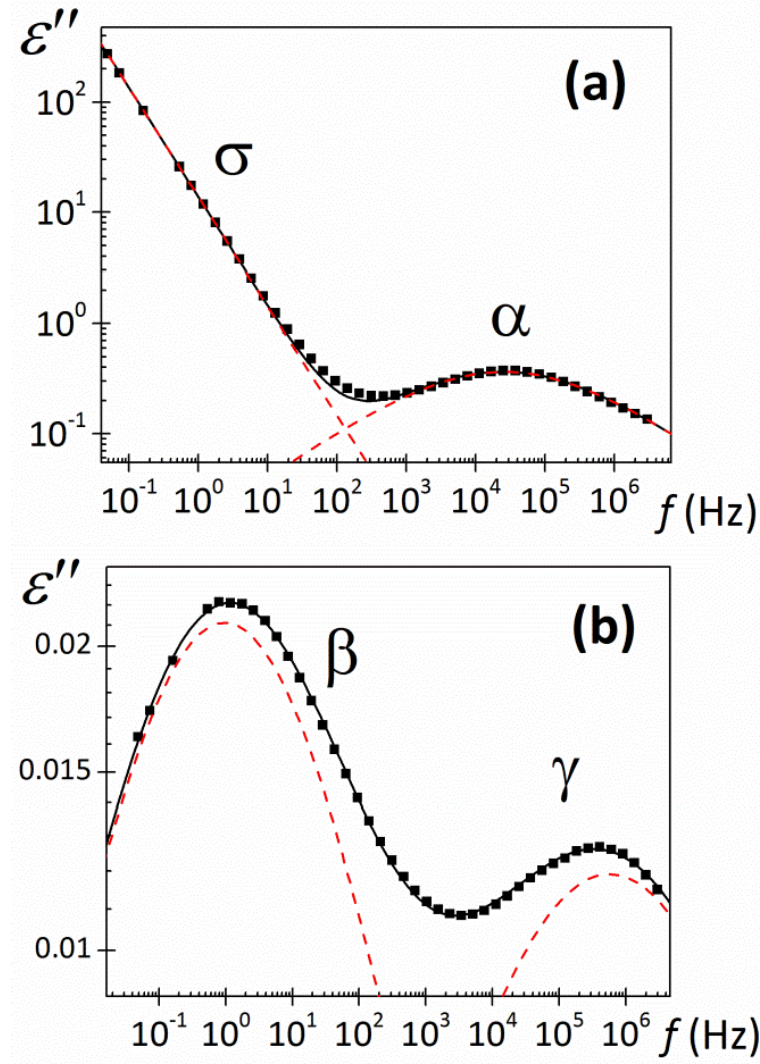

Fig. 5 Deconvolution of loss factor for 60VP/40BA-0.2C (a) conductivity and $\alpha$ processes at $348 \mathrm{~K}$ and (b) $\beta$ and $\gamma$ processes at $163 \mathrm{~K}$. Squares represent the experimental data, continuous line the HN fitting curve, and dashed lines the individual processes.

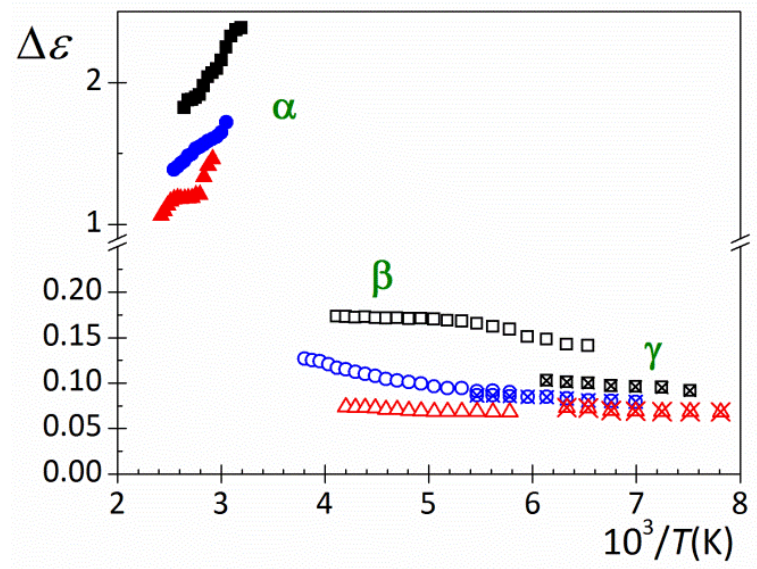

Fig. 6 Temperature dependence of the strengths of the 60VP/40BA$\mathrm{ZC}$ samples ( $\mathrm{Z}=0.2$ squares, $\mathrm{Z}=1$ circles and $\mathrm{Z}=2$ triangles) for $\alpha$ (full symbol), $\beta$ (open symbol) and $\gamma$ (cross symbol) relaxations.

On the other hand, the $\alpha$ relaxation strength decreases with both temperature and cross-linker content. This is due to the fact that the thermal energy disturbs the alignment of the molecule dipoles that intervene in the cooperative motions ${ }^{34}$ and the crosslinking produces a reduction in the molecular mobility, as observed in other polymers. ${ }^{15,51}$

Fig. 7 shows the temperature dependence of the $a_{\mathrm{HN}}$ shape parameters associated with all relaxation processes present in the $60 \mathrm{VP} / 40 \mathrm{BA}-\mathrm{ZC}$ samples $(\mathrm{Z}=0.2,1$ and 2$)$. For the $\alpha$ process the $a_{\mathrm{HN}}$ parameter increases with temperature, that is, the dielectric loss peak narrows. However, $a_{\mathrm{HN}}$ parameter decreases with the crosslinker content. The chains may experience different motions depending on their environment. Thus, the ones near the cross-link sites are in restricted environments, while those between cross-link sites will have a relaxation behaviour similar to the uncross-linked polymer. Accordingly, a higher distribution in the local environment of the single relaxing segments with cross-linking content may lead to a broadening of the relaxation. ${ }^{15}$ On the other hand, a nondefined tendency is observed in the temperature dependence of the $a_{H N}$ parameter for the $\beta$ and $\gamma$ processes.

The value of $b_{\mathrm{HN}}$ shape parameter was one at all temperatures investigated for all the processes independently of the cross-linker content. Thus, the Eq. (6) reduces to the Cole-Cole equation ${ }^{52}$ that describes a relaxation with a symmetric distribution of relaxation times. Although for amorphous polymers, a non-symmetric distribution of times is expected for the $\alpha$ relaxation, associated with macromolecular segmental motion at temperatures immediately above the $T_{g}$, symmetrical distributions have been observed for several heterogeneous systems. ${ }^{53-61}$

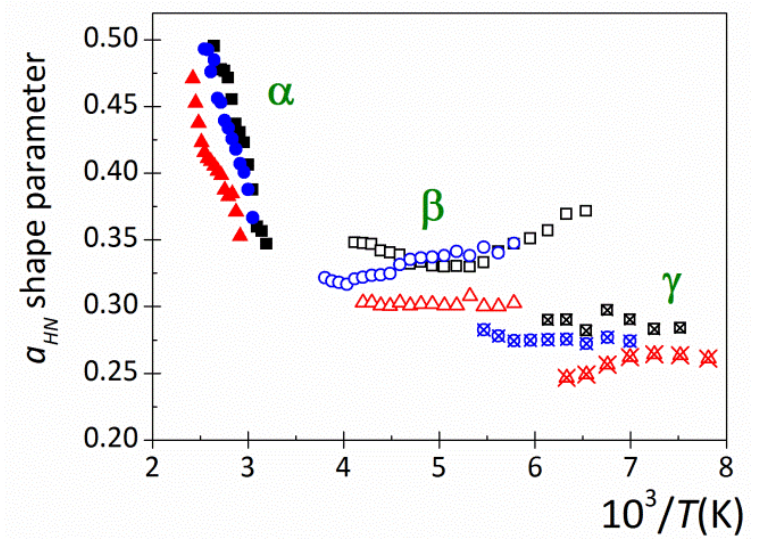

Fig. 7 Temperature dependence of the shape parameters $a_{H N}$ of the $60 \mathrm{VP} / 40 \mathrm{BA}-\mathrm{ZC}$ samples $(\mathrm{Z}=0.2$ squares, $\mathrm{Z}=1$ circles and $\mathrm{Z}=2$ triangles) for $\alpha$ (full symbol), $\beta$ (open symbol) and $\gamma$ (cross symbol) relaxations. For all processes, $b_{H N}=1$. 


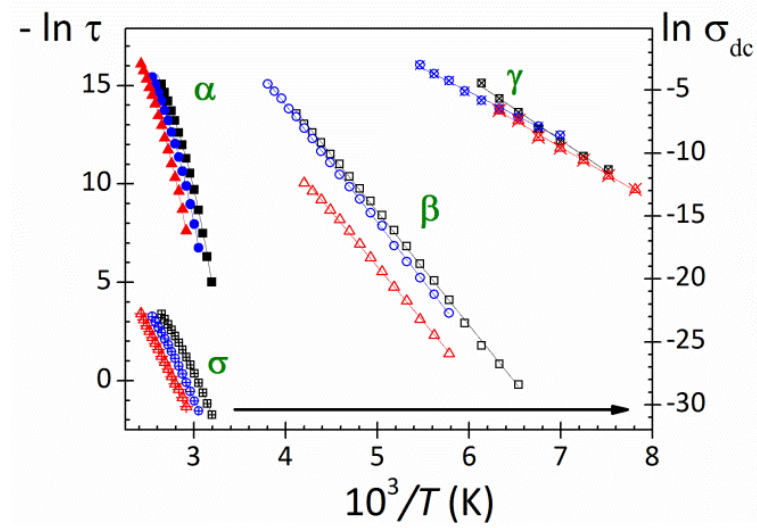

Fig. 8 Temperature dependence of the relaxation times (left- $Y$ axis) and ionic conductivity $(\sigma)$ (right- $Y$ axis), for 60VP/40BA-ZC samples with $Z=0.2$ (square), $Z=1$ (circle) and $Z=2$ (triangle). Continuous lines
represent the $v F T h$ fitting for $\alpha$ and $\sigma$ processes and the Arrhenius

fitting for $\beta$ and $\gamma$ processes.

The temperature dependence of the relaxation times, which is associated with the peak maxima of the primary and secondary absorptions, is shown in Fig. 8. According to our results, the relaxation time increases with the increase of cross-link density. This is reflected in the increase of $T_{g}$ due to the molecular motion slowdown of the polymer chains. Fig. 8 also shows the temperature dependence of dc-conductivity $\left(\sigma_{d c}\right)$. It is observed that $\sigma_{d c}$ at a constant temperature diminishes with the cross-linker content. Thus, the constraints imposed by cross-linking could hinder the diffusion of charge carriers and, consequently, the conductivity decreases.

As usual, the average relaxation time associated with the $\alpha$ relaxation is described by Vogel-Fucher-Tamman-Hesse (VFTH) equation. ${ }^{62-66}$ The deviation of the $\alpha$ relaxation from the Arrhenius behaviour may be quantified by the strength coefficient $D_{0}$ in a modified form of the VFTH equation given by

$$
\tau=A \cdot \exp \left(\frac{D_{0} \cdot T_{v}}{T-T_{v}}\right)
$$

where $A$ is a prefactor of the order of picoseconds and $T_{v}$ is the Vogel Temperature. Values of the $A, T_{v}$ and $D_{0}$ parameters that fit Eq. (8) to the experimental results are collected in Table 3. The obtained values of $T_{v}$ are nearly $50 \mathrm{~K}$ below those of $T_{g}$. Moreover, the strength coefficient obtained for these samples are lower than 10 , the frontier that separates fragile $\left(D_{0}<10\right)$ from strong $\left(D_{0}>10\right)$ glasses. ${ }^{67-69}$

The deviation from the Arrhenius behaviour can be quantified by the strength coefficient $\left(D_{0}\right)$ of Eq. (8) or by determining the slope at $T_{g}$ of the curves in the Angell plot ${ }^{35,70-72}$ (see Fig. 9), leading to the dynamic fragility index, $m$, than can be evaluated by means the followed expression:

$$
m=\frac{D_{f} \cdot T_{v}}{2.303 \cdot T_{g} \cdot\left(1-T_{v} / T_{g}\right)^{2}}
$$

Fragility values typically range between $m=16$, for strong systems, and $m=200$ for the fragile ones. ${ }^{73}$ The values of $m$, collected in Table 3 , show that, for the 60VP/40BA-ZC copolymer samples the dynamic fragility index slightly increases with the $T_{g}$. This trend is in agreement with the Angell's energy landscape model. ${ }^{67,74}$.

The apparent activation energy associated with the relaxation dynamics of the chains at $T_{g}$ can be obtained from the dynamic fragility index as $E_{\alpha}\left(T_{g}\right)=2.303 \cdot R \cdot m \cdot T_{g}$ suggesting that $E_{\alpha}\left(T_{g}\right)$ is correlated with $T_{g}$ in such a way that its value increases as $T_{g}$ goes up. Values of $E_{\alpha}\left(T_{g}\right)$ are shown in Table 3. Within the uncertainty of calculated values, it can be observed an increase of the $E_{\alpha}\left(T_{g}\right)$ with the cross-linker content.

The obtained values of $m$ and $E_{\alpha}\left(T_{g}\right)$ were found higher when comparing with the ones calculated by using the empirical expressions proposed by Qin and MEKepna ${ }^{75} 0.006 T^{\text {pqlymȩs: }}$ $m \approx 0.28( \pm 0.067) T_{g}+9( \pm 20) \quad$ and $\quad{ }_{\alpha}\left({ }_{g}\right)=\left\lceil\quad{ }_{g}()-\right.$ $\left(\mathrm{kJ} \cdot \mathrm{mol}^{-1}\right.$ ) (see Table 3). However, the same tendency in the values was observed for both methods.

On the other hand, for the $\gamma$ and $\beta$ absorptions, the temperature dependence of the relaxation times follow Arrhenius (ARRH) behaviour ( $\left.\tau_{\max }=\tau_{\infty} \cdot \exp \left[E_{a} / R T\right]\right)$. The activation energies ( $E_{a}$ ) and pre-exponential factors $\left(\tau_{\infty}\right)$ obtained are summarized in Table 3. The $\left(E_{a \gamma} / R T_{g}\right)$ is not significantly affected by the crosslinker content. However, the $\left(E_{a \beta} / R T_{g}\right)$ value is similar for the $Z=0.2$ and $Z=1$ samples, but it decreases with the highest cross-link density.

According to our previous results ${ }^{38-39}$, the slower $\beta$ relaxation for the sample with $Z=1$ is a JG process ${ }^{24,25}$ whereas the faster $\gamma$ relaxation is related to the local motions. So, the $\gamma$ and $\beta$ processes have intra- and intermolecular origin, respectively.

In order to analyse the effect of the cross-linker content on the intra/intermolecular origin of both secondary relaxation processes, we have carried out the $\mathrm{CM}$ analysis proposed for Ngai et al. ${ }^{26-30}$, which is briefly described in the introduction part. To do this, we have analysed two samples with lower $(Z=0.2)$ and higher $(Z=2)$ cross-linker content than the previously analysed $(Z=1)$. For this purpose, the normalized $\alpha$ relaxation in the time domain, $\phi(t)$, was calculated from, the retardation time spectra ${ }^{65,76}$ by using the $\mathrm{HN}$ fit parameters. From the fitting of $\phi(t)$ to the Eq. (2), the $\alpha$ characteristic relaxation time $\left(\tau^{*}\right)$ and the stretch exponent $\left(\beta_{K w w}\right)$ for the three samples analysed were obtained (see inset Fig. 9). The evolution of the $\tau^{*}$ with the temperature obeys the VFTH equation, whereas $\beta_{K w w}$ increase with temperature. This can be related to the decrease of intermolecular constraints (or coupling) between the relaxing units. On the other hand, this parameter, within the uncertainty of calculated values, is nearly independent of the crosslinker content at temperatures near $T_{g}$. So, the cross-linking does not affect significantly the strength of the intermolecular constraints on segmental relaxation at temperatures near $T_{g}$ (see Table 3). However, at higher temperatuke,s the $\beta_{K w w}$ parameter decreases with the cross-linker content ${ }^{\text {, }}$. As the cross-linking content increases, the heterogeneity is higher, so the $\beta_{k w w}$ parameter decreases. Furthermore, the temperature dependence of $\beta_{K W w}$ parameter exhibits a slope change for the $Z=2$ sample at 
high temperatures. This change could be related to the possible effect of the MWS process presence in the HN fitting of the $\alpha$ process.

According to the CM [Eq. (4) and Eq. (5)] and taking into account that (i) the $T_{g}$ is defined as the temperature at which the dielectric relaxation time $\tau^{*} \approx 100 s$ and (ii) the secondary relaxations have Arrhenius temperature dependence, the ratio $\left(E_{a}^{i} / R T_{g}\right)$, with $i=\beta$ and $\gamma$, can be evaluated as a function of $n$ and $\tau_{\infty}$ parameters as

follows: $\left.\left(\frac{E_{a}^{i}}{R T_{g}}\right)=2.303\left[(1-n) \log _{10} \tau_{\alpha}\left(T_{g}\right)+n \log _{10} t_{c}-\log _{10} \tau_{\infty i}\right]\right]^{36}$ In Table 3 are collected the values of the ratio $\left.\left(E_{a}^{i} R T\right)_{g}\right)$ evaluated by both the $\mathrm{CM}$ and the experimental data. Thus, the $\left(E_{a}^{\gamma} / R T_{g}\right)$ expt values are lower than $\left(E_{a}^{\gamma} / R T_{g}\right)_{C M}$ values for the three samples, which confirm that the $\gamma$ process has an intramolecular character. On the other hand, $\left(E_{a}^{\beta} R T\right)_{9 \text { expt }}$ value is similar to $\left(E_{a}^{\beta} R T\right)_{g \mathrm{CM}}$ value for the samples with $Z=0.2$ and 1 , but these values are different for the $Z=2$ sample. These results confirm that the $\beta$ process is a JG relaxation for $Z=0.2$ and 1 , but it changes its behaviour not for $Z=2$. Moreover, the $\left(E_{a}^{\beta} / R T_{g}\right)$ value for $\mathrm{Z}=2$ is lower than those obtained for $\mathrm{Z}=0.2$ and $\mathrm{Z}=1$.

In order to analyse the inter/intramolecular character of the $\alpha$ and $\beta$ processes the Angell plots ${ }^{74}$ are represented in Fig. 9. The increase in cross-linking only leads to a faintly increase in the cooperativity inherent to the $\alpha$ process related to the glass transition. ${ }^{15,20,78}$. Many authors have assumed a strong connection between fragility and cooperativity, ${ }^{78-82}$ with some evidence of a linear relation between them at the glass transition. In our case, both parameters do not change significantly with the cross-linker content. On the other hand, Fig. 9 evidences for the $\beta$ process differences between the samples with $Z=0.2$ and 1 , and that with $Z=2$. This behaviour can be related to the effect of the cross-linker agent on the inter/intramolecular character of the $\beta$ process, evidenced previously for the CM.

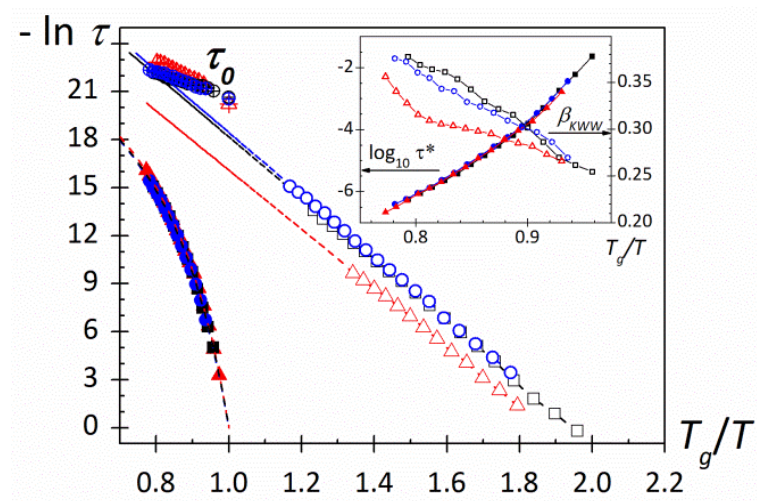

Fig. 9 Angell plot of the $\alpha$ and $\beta$ relaxations of 60VP/40BA-ZC samples with $Z=0.2$ (square), $Z=1$ (circle) and $Z=2$ (triangle). The symbols with a cross represent $\tau_{0}$ values obtained according to the $\mathrm{CM}$. Dashed lines correspond to the fitting. The glass transition temperatures used to scale the data are collected in Table 1. Inset: temperature dependence of the $\beta_{K w w}$ (open symbols) and $\tau^{*}$ (full symbols).

Table 3 Characteristic parameters of the dipolar and conductivity processes of the 60VP/40BA-ZC samples ( $\mathrm{Z}=0.2,1$ and 2 ).

\begin{tabular}{|c|c|c|c|}
\hline sample & $Z=0.2$ & $Z=1$ & $Z=2$ \\
\hline \multirow{5}{*}{ 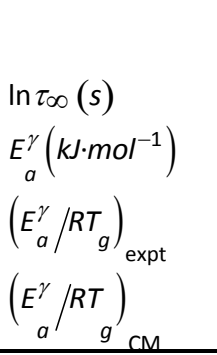 } & \multicolumn{3}{|c|}{$\gamma$ relaxation } \\
\hline & $-31.2 \pm 0.4$ & $-30.6 \pm 0.1$ & $-30.9 \pm 0.5$ \\
\hline & $22.7 \pm 1.0$ & $22.1 \pm 1.0$ & $22.6 \pm 0.5$ \\
\hline & 9.4 & 8.9 & 8.8 \\
\hline & 11.9 & 11.2 & 11.5 \\
\hline \multirow{5}{*}{$\begin{array}{c}\ln \tau_{\infty}(s) \\
E_{a}^{\beta}\left(k J \cdot m o l^{-1}\right) \\
\left.\left(E_{a}^{\beta} / R T\right)_{g}\right) \\
\left(\begin{array}{c}\left.E_{a}^{\beta} / R T\right)_{g} \\
\mathrm{~cm}\end{array}\right.\end{array}$} & \multicolumn{3}{|c|}{$\beta$ relaxation } \\
\hline & $-37.4 \pm 0.2$ & $-37.8 \pm 0.5$ & $-33.63 \pm 0.2$ \\
\hline & $47.9 \pm 0.3$ & $49.5 \pm 0.2$ & $46.3 \pm 0.6$ \\
\hline & 19.8 & 19.9 & 17.9 \\
\hline & 18.1 & 18.4 & 14.3 \\
\hline & \multicolumn{3}{|c|}{$\alpha$ relaxation } \\
\hline$-\ln A(s)$ & $25.8 \pm 0.3$ & $25.8 \pm 0.3$ & $25.9 \pm 0.4$ \\
\hline$D_{0}$ & $5.8 \pm 0.3$ & $5.9 \pm 0.2$ & $5.8 \pm 0.3$ \\
\hline$T_{v}(\mathrm{~K})$ & $244.5 \pm 2.0$ & $249.5 \pm 1.8$ & $261.1 \pm 2.0$ \\
\hline$m$ & $81.8 \pm 1.6$ & $80.4 \pm 1.3$ & $83.4 \pm 1.5$ \\
\hline$m^{*}$ & 88.9 & 90.6 & 93.6 \\
\hline$E_{a}\left(T_{g}\right)\left(\mathrm{kJ} \cdot \mathrm{mol}^{-1}\right)$ & $456 \pm 47$ & $460 \pm 40$ & $496 \pm 46$ \\
\hline$E_{a}^{*}\left(T_{g}\right)\left(\mathrm{kJ} \cdot \mathrm{mol}^{-1}\right)$ & 475 & 500 & 544 \\
\hline$\beta_{K w W}$ [at $T_{g}$ ] & 0.24 & 0.24 & 0.24 \\
\hline
\end{tabular}

The most useful feature of the $\mathrm{CM}$ is the Eq. (5), since it connects the cooperative $\alpha$ relaxation time $\left(\tau^{*} \equiv \tau_{\alpha}\right)$ with the intermolecularly uncorrelated (non-cooperative) relaxation time

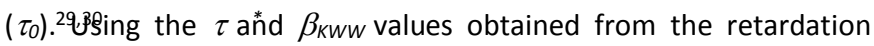
time spectra (inset Fig. 9), the $\tau_{0}$ parameter was calculated for each copolymer and for each temperature. Thus, the values of $\tau_{0}$ parameters for each sample are represented in Fig. $9 .{ }^{22,23}$ As it is observed, $\tau_{0}$ values only are superimposed to the obtained values of $\tau_{\beta}$ for $Z=0.2$ and 1 , fulfilling the Eq. (4). Hence, it is shown how $\beta$ relaxation ceases to possess typical features of pure JG relaxation for $Z=2$. 


\section{CONCLUSIONS}

This work is focused on analyzing the influence of the cross-link density on the molecular dynamics of the 60VP/40BA copolymer. The molar ratios of the ethylene glycol dimethacrylate cross-linker were varied between 0.2 and $2 \%$. As expected, the increase in cross-link density affects significantly most of the properties analysed throughout this work:

(i) The mechanical stability is enhanced.

(ii) The glass transition temperature increases due to the reduction of the segmental motion imposed by the crosslinks. The $T_{g}$ values were evaluated by MDSC and DRS techniques and a good agreement was observed between both techniques.

(iii) The $\Delta C_{p}\left(T_{g}\right)$ diminishes, related to the decrease in mobility imposed by cross-links.

(iv) Regarding to the dielectric properties, the effect on the $\alpha$ peak was: (a) a shift to higher temperatures, related to the increase of the $T_{g}$, (b) a decrease in the strength due to the reduction in the molecular mobility, (c) an increase in the breadth, as showing by the increase of $a_{H N}$ parameter, which qualitatively indicates a broader distribution of relaxation times and (d) an increase of the relaxation times due to the slowdown of the molecular motions imposed by the constraints on the polymer chains. However, only it is observed a faintly increase in the cooperativity inherent to the $\alpha$ process.

(v) The nature of the $\beta$ relaxation, including both amplitude and rate, strongly depends on the cross-linker content. According to our results, for lower cross-linker contents the $\beta$ process is a JG relaxation whereas this process ceases to have the typical behaviour of pure JG relaxation for $Z=2$. Thus, the cross-linker agent produces a decoupling between the main chain and the local chain motions. Nevertheless, molecular dynamic simulations would be useful for a definitive assignment of the origin of this relaxation.

(vi) The local motion of the polymer ( $\gamma$ relaxation) is essentially unaffected.

\section{Acknowledgements}

B.R.F, M.J.S, P.O.S and M.C gratefully acknowledge to CICYT for grant MAT2012-33483. F.G. and J.M.G. acknowledge to the Spanish Ministerio de Economía y Competitividad-FEDER (MAT2014-54137-R) and the Junta de Castilla y León (BU232U13).

\section{Notes and references}

1 V. Bühler, Polyvinylpyrrolidone Excipients for Pharmaceuticals: Povidone, Crospovidone and Copovidone; Springer: Berlin, 2005

2 F. Haaf, A. Sanner, F. Straub, Polym J 1985, 17, 143-152

3 A. Gallardo, A.R. Lemus, J. San Roman, A. Cifuentes, J.C. DiezMasa, Macromolecules 1999, 32, 610-617.

4 V. C. Grigoraş, V. Bărboui, Rev. Roum. Chim. 2008, 53, 127-131.

5 D.M. Devine, C.L. Higginbotham, Eur. Polym. J. 2005, 41, 12721279.
6 D.M. Devine, S.M. Devery, J.G. Lyons, L.M. Geever, J.E. Kennedy, C.L. Higginbotham, Int. J. Pharm. 2006, 326, 50-59.

7 S. Jin, J. Gu, Y. Shi, K. Shao, X. Yu, G. Yue, Eur. Polym. J. 2013, 49, 1871-1880.

8 M.A. Borns, S. Kalakkunnath, D.S. Kalika, V.A. Kusuma, B.D. Freeman, Polymer 2007, 48, 7316-7328.

9 N.T. Qazvini, N.Mohammadi, Polymer 2005, 46, 9088-9096.

10 W.D. Cook, T.F. Scott, S. Quay-Thevenon, J.S. Forsythe, J. Appl. Polym. Sci. 2004, 93, 1348-1359.

11 M.T. Viciosa, N. Rouzé, M. Dionísio, J.L. Gomez-Ribelles, Eur. Polym. J. 2007, 43, 1516-1529.

12 J. Jobish, N. Charoen, P. Praveen, J. Non-Crystal. Solids 2012, 358, 1113-1119.

13 S. Bekin, S. Sarmad, K. Gürkan, G. Keçeli, G. Gürdag, Sens. Actuators B Chem. 2014, 202, 878-892.

14 F. Kremer, A. Schönhals, Broadband Dielectric Spectroscopy, Springer-Verlag Berlin Heidelberg New York, 2003.

15 C.M. Roland, Macromolecules 1994, 27, 4242-4247.

16 P.N. Patil, S.K. Rath, S.K. Sharma, K. Sudarshan, P. Maheshwari, M. Patri, P. Khandelwal, P.K. Pujari, Soft Matter 2013, 9, 35893599.

17 R. Casalini, C.M. Roland, J. Polym. Sci. Part B: Polym. Phys. 2010, 48, 582-587.

18 M. Carsi, M.J. Sanchis, R. Díaz-Calleja, E. Riande, M.J.D. Nugent, Macromolecules 2012, 45, 3571-3580.

19 M. Carsi, M.J. Sanchis, R. Díaz-Calleja, E. Riande, M.J.D. Nugent, Eur. Polym. J. 2013, 49, 1495-1502.

20 S. Kalakkunnath, D.S. Kalika, H. Lin, R.D. Raharjo, B. Freeman. Macromolecules 2007, 40, 2773-2781.

21 R. Sabater i Serra, J.L. Escobar-Ivirico, J.M. Meseguer-Dueñas, A Andrio-Balado, J.L. Gomez-Ribelles, J. Polym. Sci. Part B: Polym. Phys. 2009, 47, 183-193.

22 A. Nogales, A. Sanz, T. A. Ezquerra, R. Quintana, S. MuñozGuerra Polymer 2006, 47, 7078-7084

23 A. Sanz, A. Nogales, T. A. Ezquerra, N. Lotti, L. Finelli; Phys. Review E 2004, 70, 021502

24 G.P. Johari, M. Goldstein, J. Chem. Phys. 1970, 53, 2372

25 G.P. Johari, M. Goldstein, J. Chem. Phys. 1972, 56, 4411.

26 M. Paluch, S. Pawlus, S. Hensel-Bielowka, E. Kaminska, D. Prevosto, S. Capaccioli, P.A. Rolla, ; K.L. Ngai, J. Chem. Phys. 2005, 122(23), 234506-234506-6.

27 K.L. Ngai J. Chem. Phys. 1998, 109, 6982.

28 K.L. Ngai, M. Paluch, J. Chem. Phys. 2004, 120, 857-873.

29 K.L. Ngai, S. Capaccioli, J. Phys.: Condens. Matter 2007, 19 (20), 205114.

30 R. Casalini, K.L. Ngai, C.M. Roland, Phys. Review B 2003, 68, 014201

31 K.L. Ngai, J. Phys.: Condens. Matter 2003, 15, S1107-S1125

32 K.L. Ngai, Comments Solid State Phys. 1979, 9, 141-155.

33 K.L. Ngai, K.Y. Tsang, Phys Rev E 1999, 60, 4511.

34 E. Donth, J. Polym. Sci. Part B Polym. Phys. 1996, 34(17), 28812892.

35 G. Williams, Adv. Polym. Sci. 1979, 33, 59-92.

36 K.L. Ngai, S. Capaccioli, Phys. Review E 2004, 69, 031501

37 R. Casalini, C.M. Roland, Phys. Rev. Lett. 2003, 91, 015702

38 B. Redondo-Foj, M. Carsí, P. Ortiz-Serna, M.J. Sanchis, F. García, J.M. García. J. Phys. D: Appl. Phys. 2013, 46, 295304-295315.

39 B. Redondo-Foj, M. Carsí, P. Ortiz-Serna, M.J. Sanchis, S. Vallejos, F. García, J.M. García. Macromolecules, 2014, 47(15), 5334-5346.

40 V. Bershtein, L. Egorova, P. Yakushev, P. Pissis, P. Sysel, L. Brozova, J. Polym. Sci., Part B: Polym. Phys. 2002, 40, 1056-1069.

41 N.M. Alves, J.L. Gomez-Ribelles,; J.F. Mano, Polymer 2005, 46 , 491-504.

42 T.F. Scott, W.D. Cook, J.S. Forsythe, Eur. Polym. J. 2002, 38, 705716.

43 K.W. Wagner, Arch. Electrotech. 1914, 2, 371. 
44 R.W. Sillars, Inst. Elect. Eng. 1937, 80, 378.

45 I.M. Hodge, K.L. Ngai, C.T. Moynihan J. Non-Cryst. Solids 2005, 351, 104-115

46 G. Williams, D.C. Watts, Trans. Faraday Soc. 1970, 66, 80.

47 G. Williams, D.C. Watts, S.B. Dev, A. M. North, Trans. Faraday Soc. $1971,67,1323$.

48 S. Havriliak, S. Negami, Polymer 1967, 8(4), 161-210.

49 S. Havriliak, S. Negami, J. Polym. Sci. Part B Polym. Symp. 1966, 14, 99-117.

50 S. Havriliak, S. Negami, Dielectric and Mechanical Relaxation in Materials; Hanser: Munich, 1997; p 57

51 J.K.W. Glatz-Reichenback, L. Sorriero, J.J. Fitzgerald, Macromolecules 1994, 27, 1338.

52 K.S. Cole, R.H. Cole, J. Chem. Phys. 1941, 9, 341-351.

53 R.H. Boyd, Polymer 1985, 26, 323-347.

54 E. Laredo, M. Grimau, Macromolecules 2003, 36, 9840-9850.

55 P. Huo, P. Cebe, J. Polym. Sci. Part B Polym. Phys. 1992, 30, 239250.

56 N. Noda, Polymer 2005, 46, 7201-7217.

57 Y. Ryabov, H. Nuriel, J. Polym. Sci. Part B Polym. Phys. 2003, 41(3), 217-223.

58 P. Janik, M. Paluch, Phys. Review E. 2001, 64, 042502.

59 Y. Feldman, A. Puzenko, Y. Ryabov, Chem. Phys. 2002, 284, 139168

60 P. Ortiz-Serna, R. Díaz-Calleja, M.J. Sanchis, E. Riande, R. Nunes, A. Martins, L. Visconte, Macromolecules 2010, 43, 5094-5102.

61 P. Ortiz-Serna, R. Díaz-Calleja, M.J. Sanchis, E. Riande, R. Nunes, A. Martins,; L. Visconte, J. Non-Cryst. Solids 2011, 357, 598-604.

62 E. Riande, R. Díaz-Calleja, In Electrical Properties of Polymers; Dekker, M., Ed.; The United States of America, 2004.

63 H. Vogel, Z. Phys. 1921, 22, 645-646.

64 G.S. Fulcher, J. Am. Ceram. Soc. 1925, 8, 339-340.

65 G. Tamman, W. Hesse, Z. Anorg. Allg. Chem. 1926, 156, 245247.

66 P. Lunkenheimer, U. Schneider, R. Brand, A. Loidl, Contemp. Phys. 2000, 41, 15-36.

67 C.A. Angell, Science 1995, 267, 1924-1935

68 C.A. Angell, In Complex Behavior of Glassy Systems; Proceedings of the XIV Sitges Conference, Sitges, Barcelona, Spain, 1996

69 F.J. Bermejo, H.E. Fischer, M.A. Ramos, A. de Andrés, J. Dawidowski, V. Fayos, In Complex Behaviour of Glassy Systems, ed. by M. Rubí and C. Pérez-Vicente, Springer Lecture Notes in Physics (Springer), Berlin-Heidelberg, 1997.

70 R. Bohmer, K.L. Ngai, C.A. Angell, D. Plazek, J. J. Chem. Phys. 1993, 99, 4201-4209.

71 R. Bohmer, C.A. Angell, Phys. Rev. B 1992, 45, 10091-10094.

72 R. Bohmer, C.A. Angell, Phys. Rev. B 1993, 48, 5857-5863.

73 E.G. Merino, S. Atlas, M. Raihane, A. Belfkira, M. Lahcini, A. Hult, M. Dionísio, N.T. Correia, Eur. Polym. J. 2011, 47, 1429-1446.

74 C.A. Angell, J. Non-Cryst. Solids 1991, 131-133, 13-31.

75 Q. Qin, B. McKenna, J. Non-Cryst. Solids 2006, 352, 2977.

76 F. Kohlrausch, Progg. Ann. Phys. Chem. 1854, 91, 179-214.

77 A. Reiner Zorn, J. Polym. Sci. Part B: Polym. Phys. 1999, 37, 1043.

78 C.L. Ngai, C.M. Roland, Macromolecules 1993, 26, 2688-2690.

79 K.L. Ngai, Relaxation and diffusion in complex systems, Springer:Berlin 2011.

80 M. Ikeda, M. Aniya, Intermetallics 2010, 18, 1796-1799.

81 A. Patkpwski, V. Paluch, J. Gapinski, J. Non-Cryst. Solids 2003, 330, 259-263.

82 N. Delpouve, L. Delbreilh, G. Stoclet, A. Saiter, E. Dargent, Macromolecules 2014, 47, 5186-5197. 